\title{
Multichannel Depletion-Type Field-Effect Transistor Based on Ferromagnetic Germanene
}

\author{
Jun Zheng $\odot,{ }^{1,2, *}$ Yang Xiang, ${ }^{1}$ Chunlei Li, ${ }^{3,2}$ Ruiyang Yuan, ${ }^{4}$ Feng Chi, ${ }^{5}$ and Yong Guo $\odot^{2, \dagger}$ \\ ${ }^{1}$ College of Physics Science and Technology, Bohai University, Jinzhou 121013, China \\ ${ }^{2}$ Department of Physics and State Key Laboratory of Low-Dimensional Quantum Physics, Tsinghua University, \\ Beijing 100084, China \\ ${ }^{3}$ College of Elementary Education, Capital Normal University, Beijing 100048, China \\ ${ }^{4}$ Center for Theoretical Physics, Department of Physics, Capital Normal University, Beijing 100048, China \\ ${ }^{5}$ School of Electronic and Information Engineering, University of Electronic Science and Technology of China \\ Zhongshan Institute, Zhongshan 528400, China
}

(Received 21 January 2021; revised 18 June 2021; accepted 12 July 2021; published 25 August 2021)

\begin{abstract}
Based on ferromagnetic germanene, we theoretically propose a multichannel depletion-type field-effect transistor (FET) operated by a vertical electric field. The electron transport properties of the topological transistor are investigated through the nonequilibrium Green's function method. The results reveal that new edge channels can be formed by removing some Ge atoms along the $x$ axis of the device. The proposed multichannel ferromagnetic germanene transistor has a twice on-state current and half subthreshold swing than that of traditional germanene FETs. Under the coaction of electric induced staggered potential and staggered exchange field, both the $100 \%$ spin-polarized edge states and valleys can be achieved. The ferromagnetic germanene FET can be turned off just by adjusting the electric field. In the low electric field conditions, the switching-off mechanism is due to spin blocking caused by the edge-state mismatch, while for relatively high electric field cases, both the edge-state mismatch and energy-valley mismatch are utilized. With the moderate increase in the exchange field, the switching mechanism is not changed, but the threshold electric field and breakdown voltage of the FET can be, respectively, reduced and enhanced significantly. All the results indicate that the proposed ferromagnetic germanene FET is a promising candidate for ultra-low-power dissipation topological devices.
\end{abstract}

DOI: 10.1103/PhysRevApplied.16.024046

\section{INTRODUCTION}

Germanene is a type of two-dimensional (2D) topological insulator consisting of germanium $(\mathrm{Ge})$ atoms arranged similarly to graphene and silicene in a hexagonal structure. In 1994, a decade before the successful fabrication of graphene, the structure of germanene was studied by Takeda and Shiraishi using density functional theory [1]. However, this theoretical work did not arouse widespread research interest at that time, mainly because of the common belief that strictly $2 \mathrm{D}$ materials were thermodynamically unstable and could not exist at any finite temperature $[2,3]$.

\footnotetext{
*junzheng@semi.ac.cn

†guoy66@tsinghua.edu.cn
}

Published by the American Physical Society under the terms of the Creative Commons Attribution 4.0 International license. Further distribution of this work must maintain attribution to the author(s) and the published article's title, journal citation, and DOI.
The rise of graphene triggered increased interest in other group-IV monolayers, such as silicene and germanene $[4,5]$. In 2009, the electronic properties of the planar structure of germanene was investigated by Lebegue and Eriksson. Their calculations showed that the properties related to the Dirac cone do not appear in planar germanene [6]. This conclusion was confirmed by the further theoretical research of Houssa et al. [7]. In the meantime, Cahangirov et al. [8] reported a study that answered the question of whether a stable freestanding 2D structure can be constructed from $s p^{2}$-like hybridized germanium atoms. The calculation of the electronic structure showed that germanene consists of both $s p^{2}$ and $s p^{3}$ bonds, in which $s p^{2}$ hybridization causes it to exhibit a Dirac electronic structure similar to graphene. In addition, germanene with a low-buckled honeycomb structure is more stable than that with high-buckled structure. In 2011, the theoretical study of Yao et al. [9] confirmed that germanene tends to form a low-buckled structure rather than a planar one, and Ge atoms with a 2D honeycomb geometry have nontrivial topological properties in their native structure. 
Due to the buckled structure, the germanene lattice is decomposed into two vertically displaced sublattices. When a vertical electric field is applied, the atoms in different sublattices are no longer equivalent, and the inversion symmetry is broken. As a consequence, a tunable direct band gap can be opened at the Dirac point [1014]. Based on these properties, a variety of silicene and germanene field-effect topological transistors have been proposed based on theory [15-21]. On the experimental side, a growing number of groups have successfully synthesized germanene on conductor and semiconductor substrates since 2014 [22-26]. Although freestanding germanene has yet to be synthesized, single-layer silicene and germanene FETs have now been fabricated successfully $[27,28]$.

Topological insulators are characterized by a full insulating bulk gap and time-reversal symmetry-protected gapless edge or surface states. The nondissipation transport properties of the edge or surface state make the topological device a promising candidate for ultra-low-power consumption electronics $[29,30]$. However, the on-state current of a topological transistor, which using edge states for information transport is much smaller at low bias voltages compared with the traditional depletion-type FET. It is generally recognized that a small on-state current increases the delays of transistors, thus limiting large-scale integration applications [31].

In this paper, we propose a multichannel ferromagnetic (FM) germanene FET with a relatively high on-state current and low threshold parameter at low bias voltages. As shown in Fig. 1, the central device is divided into two regions, and the germanene in each region is sandwiched between two ferromagnetic insulators with opposite magnetization. Some Ge atoms in the germanene nanoribbon are removed along the $x$ axis. The dual gates are placed above and below the dielectric layers to control the electric field perpendicular to the sheet. It should be pointed out that even though its structure is similar to that of a normal germanene FET, which requires only band gaps to turn the transistor off, the fundamental switching mechanism of the FM germanene FET is completely different.

\section{MODEL AND METHODS}

The source and drain electrode and the central device of the FM germanene transistor can be expressed by the tight-binding Hamiltonian [32-35]:

$H_{S}=H_{D}=-t \sum_{<i j>, \sigma} c_{i \sigma}^{\dagger} c_{j \sigma}+i \frac{\lambda_{\mathrm{SO}}}{3 \sqrt{3}} \sum_{\ll i j \gg, \sigma \bar{\sigma}} v_{i j} c_{i \sigma}^{\dagger} s_{\sigma \bar{\sigma}}^{z} c_{j \bar{\sigma}}$

where the index $\langle i j\rangle(\ll i j \gg)$ means that the summation is extended over all the (next-) nearest-neighbor

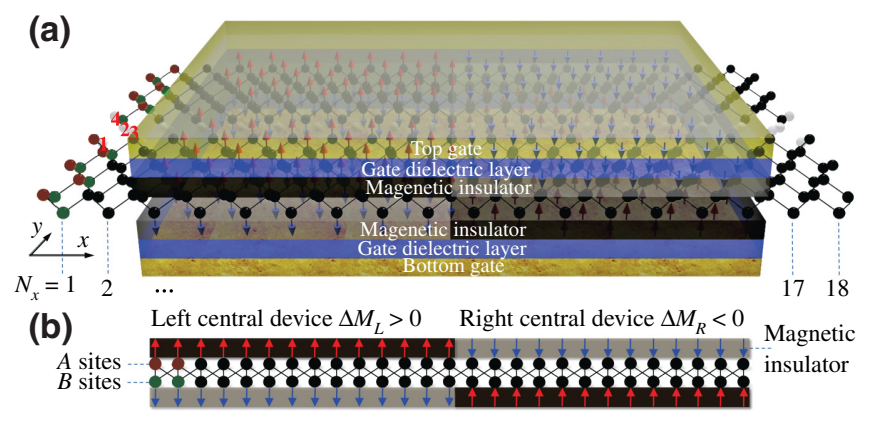

FIG. 1. (a) Schematic cross-section view of the ferromagnetic germanene FET, in which the central device is decomposed into seven layers. The Ge atoms (represented by the black spheres) form a buckled germanene layer, and the unit cell of the germanene is marked by red spheres ( $A$ sites) and green spheres ( $B$ sites). The number of unit cells in the $x$-axis direction and atom sites (black and light gray spheres) in per unit cell are $N_{x}=18$ and $N_{y}=24$, respectively. Some Ge atoms in the germanene sheet with $N_{x}=18$ and $N_{y}=4$ are removed. The removed Ge atoms are represented by light gray spheres, and four Ge atoms removed along the $y$ axis on the leftmost side are marked with red numbers. A pair of top and bottom gates are used to provide an electric field perpendicular to the germanene. (b) Side schematic of the germanene and magnetic insulator layers. Each magnetic insulator layer is divided into two parts, and the magnetization of the magnetic insulator is indicated by the red arrows in the dark region and blue arrows in the light region.

sites. The spin-up and spin-down electrons are distinguished with the spin index $\sigma=-\bar{\sigma}= \pm 1$. The first term in Eq. (1) represents the nearest-neighbor hopping between sublattices with the transfer energy $t$. The second term describes the effective spin-orbit coupling with strength $\lambda_{\text {So }}$, and the site-dependent Haldane phase factor $v_{i j}=+1$ $\left(v_{i j}=-1\right)$ if the next-nearest-neighbor hopping is anticlockwise (clockwise) around the positive $z$ axis [36,37]. $s^{z}$ is the $z$ component of the Pauli matrix.

$$
\begin{aligned}
H_{C}= & H_{S}+\lambda_{E} \sum_{i, \sigma} \mu_{i} c_{i \sigma}^{\dagger} c_{i \sigma} \\
& +M \sum_{i \in L, \sigma} \mu_{i} c_{i \sigma}^{\dagger} s_{\sigma \sigma}^{z} c_{i \sigma}-M \sum_{i \in R, \sigma} \mu_{i} c_{i \sigma}^{\dagger} s_{\sigma \sigma}^{z} c_{i \sigma},
\end{aligned}
$$

where the second term represents the staggered potential induced by the electric field with strength $\lambda_{E}$ and $\mu_{i}=+1(-1)$ for the $A(B)$ sites $[11,38]$. The strength of $\lambda_{E}$ can be controlled by applying a $z$-directed electric field $E_{z}(x, y)$ with $\lambda_{E}=\ell E_{z}(x, y)$, in which $\ell=$ $0.33 \AA$ is the sublattice separation distance [9]. The vertical electric field applied to the germanene can be written as $E_{z}(x, y)=\left(V_{t}-V_{b}\right) /\left[d_{0}-2\left(d_{D}+d_{M}\right)+2\left(d_{D}+\right.\right.$ $\left.\left.d_{M}\right) /\left(\varepsilon_{D}+\varepsilon_{M}\right)\right]$ [15], where $V_{t}$ and $V_{b}$ are top gate and bottom gate voltages, $d_{0}$ is the distance between the two gates, $d_{D}$ and $d_{M}$ are the thickness of the dielectric and 
magnetic insulator regions, $\varepsilon_{D}$ and $\varepsilon_{M}$ are the dielectric constants of the dielectric layer and magnetic insulator layer, respectively. The corresponding total gate voltage is $V_{g}=V_{t}+V_{b}$.

The last two terms describe the exchange fields of different directions with strength $M$ operating on the $\mathrm{Ge}$ atoms at $A\left(\mu_{i}=+1\right)$ and $B\left(\mu_{i}=-1\right)$ sublattices in the left $(i \in L)$ and right $(i \in R)$ central device regions. As proposed for graphene and silicene, a large exchange field could be induced in germanene by the magnetic proximity effect with a magnetic insulator such as $\mathrm{EuO}$ [39-41]. In the simulation calculation, the magnetization of adjacent ferromagnets is set to be opposite, the staggered exchange field applied to the left (right) central device region is $\Delta M_{L}=M_{L}^{A}-M_{L}^{B}=2 M\left(\Delta M_{R}=M_{R}^{A}-\right.$ $\left.M_{R}^{B}=-2 M\right)$ [42]. In addition, four $\mathrm{Ge}$ atoms around $N_{y} / 2$ in the device are removed along the $x$ direction. Hence, in the following calculation, $i(j) \notin\left\{N_{y} / 2, N_{y} / 2 \pm\right.$ $\left.1, N_{y} / 2+2\right\}$. Due to the edge states are protected from the effects of weak electron interactions $[43,44]$, and we focus on the influence of electric and exchange fields in this paper, the electron-electron interaction is neglected.

Within the Landauer-Büttiker formalism, the current flowing through the drain electrode can be expressed as $[45,46]$

$$
I_{D}=\frac{e}{h} \sum_{\sigma} \int T_{\sigma}(\varepsilon)\left[f_{S}(\varepsilon)-f_{D}(\varepsilon)\right] d \varepsilon
$$

where $T_{\sigma}(\varepsilon)$ is the spin-dependent transmission coefficient. Without local external fields, the transmission coefficient satisfies $T_{\sigma}=T_{S D \sigma}=T_{D S \sigma}$, where $T_{S D \sigma}=$ $\operatorname{Tr}\left[\Gamma_{S \sigma} \mathbf{G}_{\sigma}^{r} \Gamma_{D \sigma} \mathbf{G}_{\sigma}^{a}\right]$ is related to the electron transport from the source to the drain electrode with the linewidth function $\Gamma_{S(D) \sigma}=i\left(\Sigma_{S(D) \sigma}^{r}-\Sigma_{S(D) \sigma}^{a}\right)$. The advanced and retarded self-energy function of the $\alpha$ lead can be obtained from $\Sigma_{\alpha \sigma}^{a(r)}=\mathbf{H}_{C \alpha} \mathbf{g}_{\alpha \sigma}^{a(r)} \mathbf{H}_{\alpha C}$, and the Hamiltonian matrix $\mathbf{H}_{C \alpha}$ describes the coupling between the central device and $\alpha$ lead. The surface Green's function $\mathbf{g}_{\alpha \sigma}^{r(a)}$ can be calculated by the Green's function method or transfer matrix [47,48]. The Green's function of the entire system is given by $\mathbf{G}_{\sigma}^{r}(\varepsilon)=\left[\varepsilon \mathbf{I}-\mathbf{H}_{0}-\right.$ $\left.\Sigma_{S \sigma}^{r}-\Sigma_{D \sigma}^{r}\right]^{-1}=\left[\mathbf{G}_{\sigma}^{a}(\varepsilon)\right]^{\dagger}$, in which $\mathbf{H}_{0}$ is the Hamiltonian matrix of the central device. The Fermi function $f_{\alpha}(\varepsilon)=\left[e^{\left(\varepsilon-\mu_{\alpha}\right) / k_{B} T}+1\right]^{-1}$, where $T$ is the system equilibrium temperature, which is set to $4.2 \mathrm{~K} . \mu_{\alpha}$ is the electrochemical potential of the $\alpha$ electrode. For a given bias voltage $V_{D S}$, the electrochemical potentials of the electrodes are fixed at $\mu_{S}=e V_{S}=e V_{D S} / 2$ and $\mu_{D}=e V_{D}=$ $-e V_{D S} / 2$.

For the general tight-binding Hamiltonian $H=\sum_{i \sigma, j \bar{\sigma}}$ $c_{i \sigma}^{\dagger} \mathbf{H}_{i \sigma, j \bar{\sigma}} c_{j \bar{\sigma}}$, the local current flowing from a site $i$ with spin $\sigma$ under a dc bias can be expressed as [49-54]

$$
J_{i \sigma}=\sum_{j \bar{\sigma}} J_{i \sigma, j \bar{\sigma}}=\frac{e}{h} \sum_{j \bar{\sigma}} \int \operatorname{Re}\left[H_{i \sigma, j \bar{\sigma}} G_{j \bar{\sigma}, i \sigma}^{<}(\varepsilon)\right] d \varepsilon .
$$

When the device is under a small bias and at zero temperature, by applying the Keldysh equation $G^{<}(\varepsilon)=$ $G^{r}(\varepsilon)\left[i \Gamma_{S} f_{S}(\varepsilon)+i \Gamma_{D} f_{D}(\varepsilon)\right] G^{a}(\varepsilon)$ and the electron correlation function $G^{n}(\varepsilon)=G^{r}(\varepsilon) \Gamma_{S} G^{a}(\varepsilon)$ [55], the spindependent nonequilibrium local current can be written as

$$
J_{i \sigma, j \bar{\sigma}}=\frac{2 e^{2}}{h} \operatorname{Im}\left[H_{i \sigma, j \bar{\sigma}} G_{j \bar{\sigma}, i \sigma}^{n}(\varepsilon)\right]\left(V_{S}-V_{D}\right)
$$

\section{RESULTS AND DISCUSSION}

In the numerical results presented below, the size of the central device region is fixed as $N_{x}=80$ and $N_{y}=84$. The nearest-neighbor hopping energy $t$ and effective spin-orbit coupling strength $\lambda_{\text {SO }}$ of germanene are taken as $t=1.3$ $\mathrm{eV}$ and $\lambda_{\mathrm{SO}}=43 \mathrm{meV}$ [56]. The strength of the exchange field induced by the magnetic proximity effect is fixed as $M=\lambda_{\mathrm{SO}} / 2$, the corresponding staggered exchange fields acting on the left and right sides of the central device are $\Delta M_{L}=2 M=\lambda_{\text {SO }}$ and $\Delta M_{R}=-2 M=-\lambda_{\text {SO }}$. In the simulation calculation of the drain current $I_{D}$ (local current $\left.J_{i \sigma, j \bar{\sigma}}\right)$, the system equilibrium temperature is set to $4.2 \mathrm{~K}$ $(0 \mathrm{~K})$. The conversion between the current units $e / h$ and $\mu \mathrm{A}$ is $1 e / h=38.74 \mu \mathrm{A}$.

The drain currents $I_{D}$ of the multichannel ferromagnetic FET as a function of the electric-field-induced staggered potential $\lambda_{E}$ for bias voltages of $V_{D S}=10,30$, and $50 \mathrm{mV}$ are plotted in Fig. 2(a). As a comparison, under the same bias voltage, Figs. 2(b) and 2(c) show the drain current of the dual-channel and multichannel traditional germanene FET. It can be seen from the figures that multichannel can effectively increase the on-state current, but traditional transistors still need a strong electric field to cut off the current.

As shown in Fig. 2(a), the multichannel ferromagnetic germanene FET has two advantages over the traditional dual-channel FET: (i) a larger on-state current and lower subthreshold swing and (ii) a lower threshold staggered potential.

When $\lambda_{E}=0$, the on-state current of the multichannel FET [see Fig. 2(a)] can be up to twice that of the dualchannel germanene FET [see Fig. 2(b)]. This is because, for the traditional dual-channel FET with the local current density distribution shown in the inset of Fig. 2(b), only two boundary channels are involved in the current transport, in which the spin-down (spin-up) current transfers from the source to the drain electrode through the upper (lower) edge. However, as shown in Fig. 2(d), if some Ge atoms located at hollow gray circles are removed, 

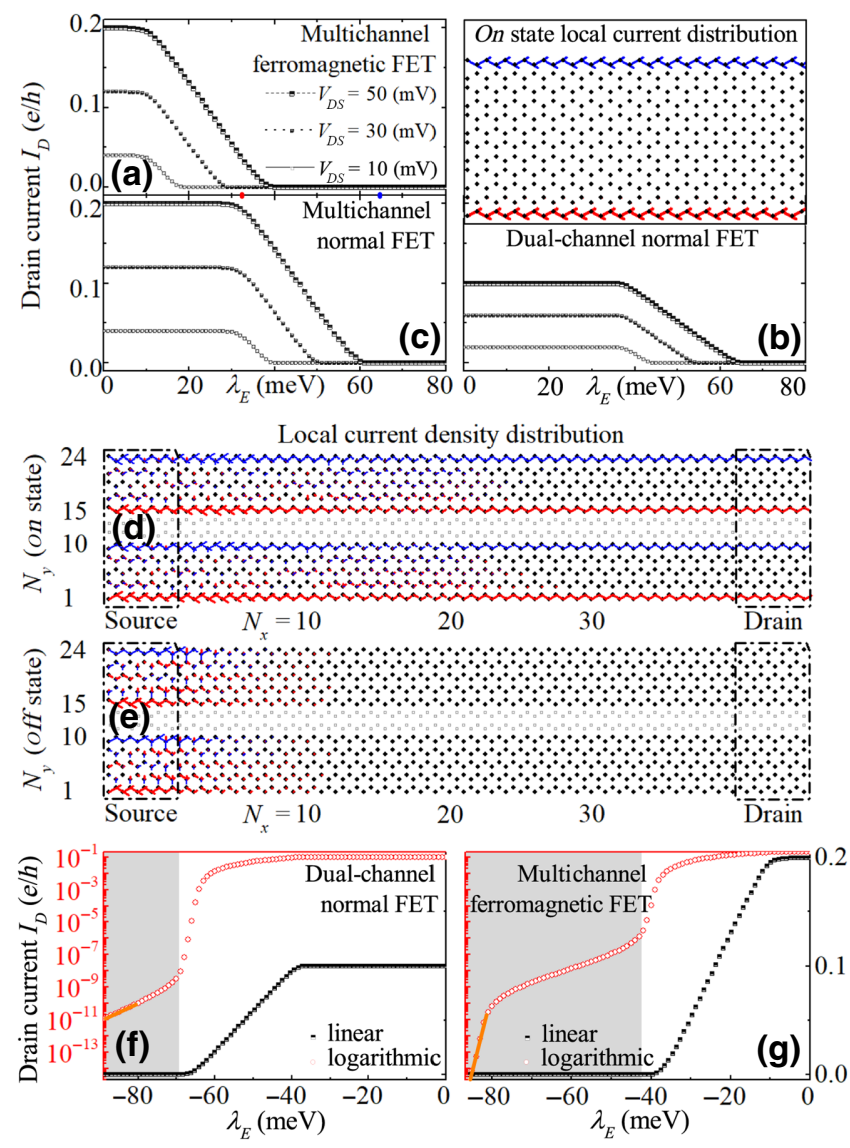

FIG. 2. (a) Drain current $I_{D}$ of the multichannel ferromagnetic germanene FET as a function of electric-field-induced staggered potential $\lambda_{E}$ with different values of the bias voltage $V_{D S}$. (b) Drain current $I_{D}$ of the dual-channel normal germanene FET versus $\lambda_{E}$ with bias voltages of $V_{D S}=10,30$, and $50 \mathrm{mV}$. Inset shows the corresponding on-state local current density distribution with $\lambda_{E}=0$ and the energy $\varepsilon=10 \mathrm{meV}$. (c) Drain current $I_{D}$ of the multichannel normal germanene FET as a function of $\lambda_{E}$ with bias voltages of $V_{D S}=10,30$, and $50 \mathrm{mV}$. (d) Local current density distribution of multichannel germanene transistor in the on state with $\lambda_{E}=0$ and $\varepsilon=10 \mathrm{meV}$, and with central region size $N_{x}=40$ and $N_{y}=24$. The thick red (thin blue) arrow corresponds to the spin-up (spin-down) current. The length and direction of the arrow represent the magnitude and direction of the current density vector at each lattice site. The black solid circle represents the position of the Ge atom, and the gray hollow circle corresponds to the location of the removed Ge atom. (e) Local current density distribution of multichannel transistor in the off state with $\lambda_{E}=\lambda_{\mathrm{SO}} / 2$, and the other parameters are the same as those in Fig. 2(d). Logarithmic and linear current of the dual-channel normal germanene FET (f) and the multichannel ferromagnetic germanene FET $(\mathrm{g})$ versus $\lambda_{E}$ with bias voltage $V_{D S}=50 \mathrm{mV}$.

new edge channels with opposite spin will appear at the critical interface. Therefore, under a small forward bias, the spin-up and down-spin currents can flow to the drain electrode mainly through four edge channels. Figures 2(f) and $2(\mathrm{~g})$ depict the logarithmic and linear current of the dual-channel normal germanene FET and the multichannel ferromagnetic germanene FET versus the electric-fieldinduced staggered potential. By comparing two $\log \left(I_{D}\right)$ plots, the subthreshold slope (the reciprocal value of the subthreshold swing) of the multichannel ferromagnetic FET in the relatively high-electric-field region is nearly doubled than that of dual-channel normal germanene FET [see the orange solid lines in Figs. 2(f) and 2(g)]. There are two steep slopes in the $\log \left(I_{D}\right)-\lambda_{E}$ plot as shown by the red circles in the gray area of Fig. $2(\mathrm{~g})$, the larger and smaller sloped regions correspond to different current cutoff mechanisms. In the larger slope region ( $\lambda_{E}$ around $\left.2 \lambda_{S O}\right)$, both the energy-band mismatch and two valley gaps are used to cut off the drain current. However, in the smaller slope region, only the energy-band mismatch is used. In addition, the current cutoff of the dual-channel normal germanene FET utilizes only the band gap opened by the electric field, and hence the $\log \left(I_{D}\right)-\lambda_{E}$ plot in the gray area of Fig. 2(f) only has one steep slope.

As shown in Fig. 2(b), for a dual-channel normal germanene FET, the output current $I_{D}$ can reach about zero only when the staggered potential satisfies $\lambda_{E}>\lambda_{\text {SO }}+$ $V_{D S} / 2$. However, with the aid of the magnetic proximity effect, the FM germanene FET can be turned off with $\lambda_{E}>\lambda_{\text {SO }}-M+V_{D S} / 2$. A much lower threshold staggered potential means not only lower power consumption but also a different switch-off mechanism. When the FM germanene transistor is in the off state, there are still a small number of spin-up electrons that can propagate through the edge into the device region near the source lead [see Fig. 2(e)]. This indicates that the germanene under a weak electric field is not transformed into a topological trivial insulator. Hence, the FM germanene FET does not utilize the band gap to block the current.

To understand the switching mechanism and transport properties, the energy band and corresponding conductance diagrams of the normal and FM germanene are given in Figs. 3 and 4. As presented in Figs. 3(a) and 3(d), without considering the effect of the external field, the energy levels of the edge states degenerate and cross at the $\Gamma$ $\left(k_{x a}=\pi\right)$ point. Moreover, because some of the Ge atoms are removed along the $x$ axis in the real space in the calculation, a pair of new edge states with opposite spins is formed in the bulk band-gap region, and the degeneracy of the states is 4 . When a relatively low staggered exchange field is applied $\left(\left|\Delta M_{L(R)}\right|=2 M<2 \lambda_{\text {So }}\right)$, the width of the bulk band gap in the $K$ valley ( $K^{\prime}$ valley) becomes smaller (larger), but the edge state is not destroyed, and its degeneracy is still 4 [see Figs. 3(b) and 3(c)]. Under the condition of a small positive bias and considering the spinmomentum locking, electrons can flow from the left to the right electrode through two spin-up and two spin-down edge states [see Fig. 2(d)]. 


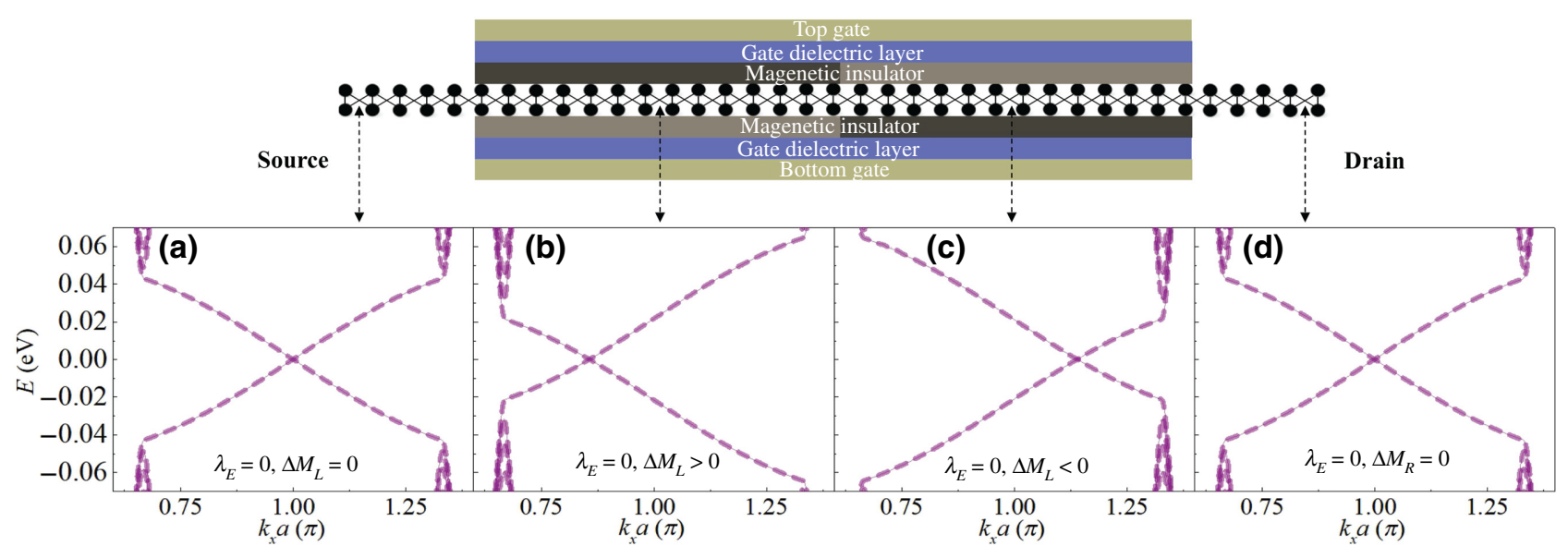

FIG. 3. Energy-band diagrams of (a) the source electrode with $\lambda_{E}=0$ and $\Delta M_{L}=0$; (b) the left region of the central device with $\lambda_{E}=0$ and $\Delta M_{L}=\lambda_{\mathrm{SO}}$; (c) the right region of the central device $\lambda_{E}=0$ and $\Delta M_{R}=-\lambda_{\mathrm{SO}}$; (d) the drain electrode with $\lambda_{E}=0$ and $\Delta M_{R}=0$. The purple lines represent spin-degenerate energy states.

It should be pointed out that from the comparison of Figs. 3(a) and 3(b), Figs. 3(b) and 3(c), Figs. 3(c) and $3(d)$, the slopes of edge states are different, which means that the velocities of electrons in the electrodes and the left and right central device regions are different. Therefore, as shown in Fig. 2(d), when the current flows from the source to the drain electrode, there will be internal current due to interface scattering. However, due to the degree of edge states mismatch is low, electrons transport to the drain electrode mainly through the edge channels, and the internal current is much smaller than the edge current.

The staggered electric potential and the staggered exchange field can break the time-reversal symmetry and the spin rotation symmetry. Under the combined action of staggered electric potential and staggered exchange field, and if $\lambda_{E}+M>\lambda_{\text {SO }}$, the edge states can transform from a quantum spin Hall state to the spin-polarized quantum anomalous Hall state, and the $K$ and $K^{\prime}$ valleys
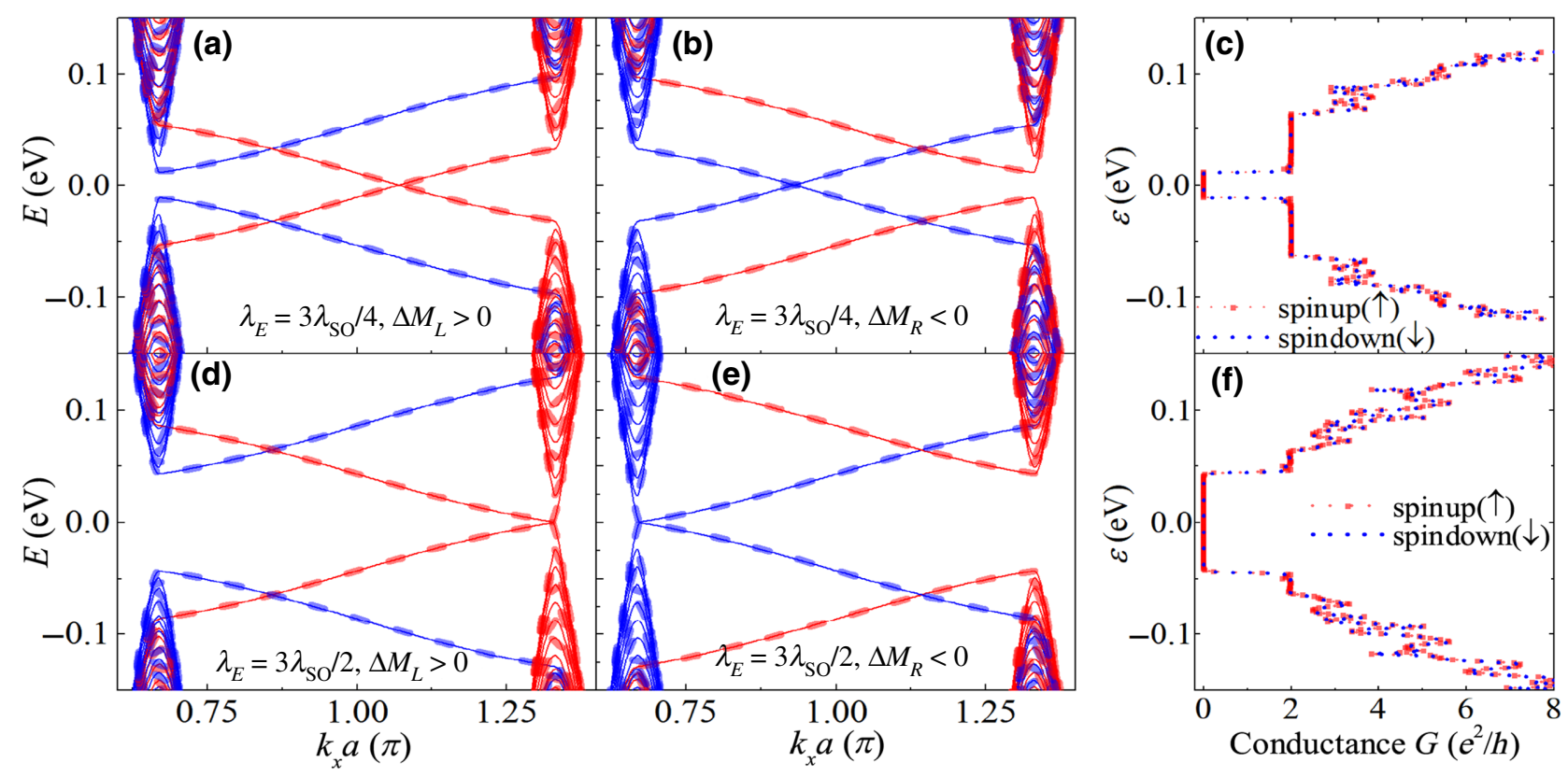

FIG. 4. Energy-band diagrams of germanene with different values of the electric-field-induced potential and the staggered exchange field: (a) $\lambda_{E}=3 \lambda_{\mathrm{SO}} / 4, \Delta M_{L}=\lambda_{\mathrm{SO}}$; (b) $\lambda_{E}=3 \lambda_{\mathrm{SO}} / 4, \Delta M_{R}=-\lambda_{\mathrm{SO}}$; (d) $\lambda_{E}=3 \lambda_{\mathrm{SO}} / 2, \Delta M_{L}=\lambda_{\mathrm{SO}}$; (e) $\lambda_{E}=3 \lambda_{\mathrm{SO}} / 2, \Delta M_{R}=$ $-\lambda_{\mathrm{SO}}$. The red and blue lines represent spin-up and spin-down energy states, respectively. (c) Conductance of ferromagnetic (FM) germanene as a function of the energy $\varepsilon$ with $\lambda_{E}=3 \lambda_{\mathrm{SO}} / 4$ and $\Delta M_{L(R)}= \pm \lambda_{\mathrm{SO}}$. (f) Conductance $G$ versus the energy $\varepsilon$ with $\lambda_{E}=3 \lambda_{\mathrm{SO}} / 2$ and $\Delta M_{L(R)}= \pm \lambda_{\mathrm{SO}}$. 
are $+100 \%$ or $-100 \%$ spin polarized in different energy ranges. As illustrated in Fig. 4(a) [Fig. 4(b)] for $\Delta M_{L}>0$ $\left(\Delta M_{R}<0\right)$, spin-down (spin-up) edge states are destroyed and lead to a direct band gap of $2\left|\lambda_{\text {SO }}-\right| \lambda_{E}|-M|$ at the $K\left(K^{\prime}\right)$ point. The spin directions of the undestroyed edge states in Figs. 4(a) and 4(b) are opposite, and the edge-state mismatch between the left and right parts of the device gives rise to zero conductance within the corresponding energy window [see Fig. 4(c)]. The strength of electric-field-induced staggered potential in Figs. 4(a)-4(c) is $\lambda_{E}=3 \lambda_{\mathrm{SO}} / 4$, the corresponding $x$-axis coordinate $\lambda_{E}=$ $3 \lambda_{\mathrm{SO}} / 4=32.25 \mathrm{meV}$ displayed in Fig. 2(a) is marked with a red dot. It can be seen from Fig. 2(a) that under the condition of a weak electric field, only using the edge-state mismatch cannot cutoff the current generated by relatively large bias.

Figures 4(d) and 4(e) show the energy bands of FM germanene with the same intensity exchange fields as in Figs. 4(a) and 4(b), but a higher electric-field-induced staggered potential $\lambda_{E}=3 \lambda_{\mathrm{SO}} / 2=64.5 \mathrm{meV}$, which is marked with a blue dot in the $x$-axis coordinate of Fig. 2(a). When the staggered exchange field $\Delta M_{L}>0\left(\Delta M_{R}<0\right)$, the band gap of the spin-down (spin-up) edge states in the $K$-valley ( $K^{\prime}$-valley) region increases with the strength of the electric field. At the same time, the spin-up (spin-down) $K^{\prime}$ valley ( $K$ valley) moves toward the Fermi level, and the energy valley shows $100 \%$ spin polarization, as shown by the red (blue) thin solid line and thick dashed line in Fig. 4(d) [Fig. 4(e)]. Because of the mismatch between the energy bands of the edge states and the energy valleys in the $\Delta M_{L}>0$ and $\Delta M_{R}<0$ regions, the conductive paths can be blocked in a relatively larger energy range [see Fig. 4(f)]. It is worth note that if the magnetic proximity effect is not considered, a larger electric field $\lambda_{E}=2 \lambda_{\text {SO }}$ is needed to make germanene produce a large enough band gap to achieve the same degree of conductance suppression, which is shown in Fig. 4(f). In addition, the subthreshold slope of the dual-channel normal germanene FET and the multichannel ferromagnetic germanene FET is different, due to the switching-off mechanisms of the two transistors are completely different. The dual-channel normal germanene FET requires a large electric field to open a band gap, while the multichannel ferromagnetic germanene FET mainly uses energy-band mismatch. When the energy-valley mismatch and the $K$-valley and $K^{\prime}$ valley gaps are used to turn off the transistor, the subthreshold slope of the multichannel ferromagnetic germanene FET can be nearly double that of the dual-channel normal germanene FET.

As the magnetic proximity effects of different ferromagnetic materials have been studied in previous theoretical work, we next discuss the influence of the exchange field strength on the off -state threshold parameter and breakdown voltage of the device. Figures 5(a)-5(c) depict the drain current $I_{D}$ as a function of the electric-field-induced

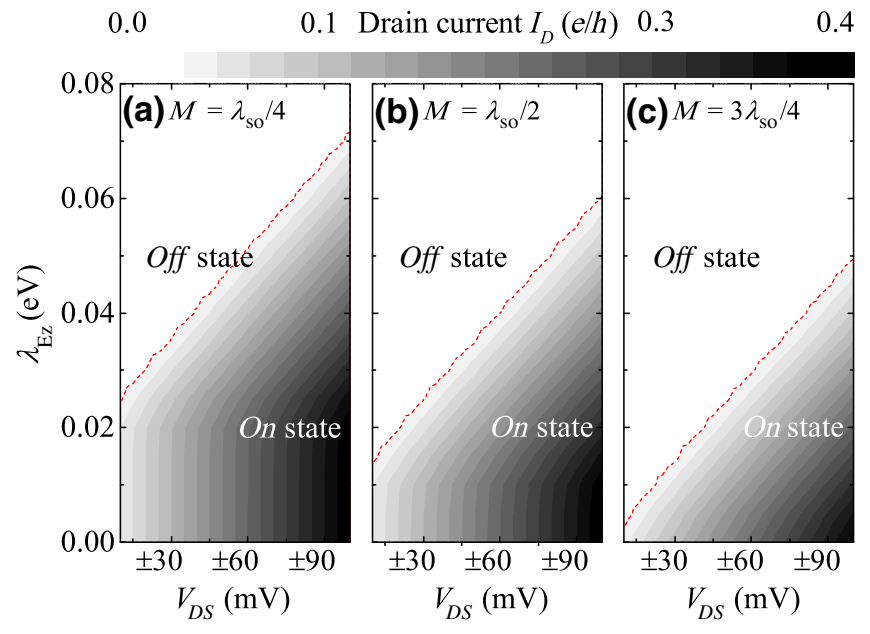

FIG. 5. Drain current $I_{D}$ of the multichannel FM germanene transistor for different values of the electric-field-induced staggered potential $\lambda_{E}$ and bias voltage $V_{D S}$ for (a) $M=\lambda_{\mathrm{SO}} / 4$, (b) $M=\lambda_{\mathrm{SO}} / 2$, and (c) $M=3 \lambda_{\mathrm{So}} / 4$.

staggered potential $\lambda_{E}$ and bias voltage $V_{D S}$ for different values of the exchange field $M$. It can be seen from the figure that whether the intensity of the exchange field is weak or strong, the FM germanene transistor can be turned on and off by adjusting the $z$-axis electric field. In the on state with $\lambda_{E}=0$, because the current in the low biasvoltage regime is transported only through the edge state, the corresponding current values are the same under different $M$. However, in the case of a high bias voltage, the current intensity increases with the increase in $M$ due to the bulk energy bands participating in the electron transport [see the black regions in Figs. 5(a)-5(c)]. In addition, the numerical simulation results show that with the increase in the exchange field, the threshold electric field strength of the off state will decrease, and the breakdown voltage the device can withstand will increase significantly under the same electric field [see the white regions in Figs. 5(a)-5(c)].

Finally, we briefly discuss the effect of edge hydrogenation and random impurities on the numerical simulation results. In a realistic germanene nanoribbon, there will be a dangling bond at the edge. Usually, the Ge atoms in the boundary are passivated by hydrogen to improve the stability of zigzag germanene nanoribbons. When both the zigzag edges are fully $\mathrm{Ge}-\mathrm{H}$ bonded, the characteristic edge states of germanene are completely preserved, the $\mathrm{H}$ edge-hydrogenated zigzag germanene nanoribbon behaves like a bare freestanding germanene nanoribbon. When the edges of germanene are $\mathrm{Ge}-\mathrm{H} 2$ bonded, however, the edge states are destroyed and an energy gap is opened at the Fermi level, the H2-edge-hydrogenated zigzag germanene nanoribbon behaves like a $2 \mathrm{D}$ semiconductor $[57,58]$. Since the on state of the topological transistor, which we study in the paper, mainly uses the edge state 
to conduct the current, and the transistor in the off state is mainly utilize the edge-state mismatch. Therefore, when the Ge atoms at the edge are passivated by one hydrogen atom, all results in the paper can also be obtained. In addition, real materials always have random impurities. The time-reversal symmetry-protected edge states could be destroyed when magnetic impurities are introduced into the system. Due to there being more edge states carrying the current in multichannel FET than in dual-channel FET, the magnetic impurities have a more obvious influence on the multichannel FET. However, since nonmagnetic impurities do not break the time-reversal symmetry, the helical property of the edge states is topologically protected, both the multichannel FET and dual-channel FET are robust in the presence of nonmagnetic impurities.

\section{CONCLUSIONS}

In summary, we theoretically study the electron transport properties of a multichannel germanene transistor, in which the FM-sandwiched germanene is subjected to a $z$-directed electric field. It is found that the transistor considered can be switched between on and off states by tuning only the strength of the electric field. Compared with the normal germanene FET, the multichannel FM germanene FET has the following main features. (1) When Ge atoms are removed along the $x$ axis, two new edge channels with opposite spin are created at the boundary, the on state current of the multichannel germanene FET at $\lambda_{E}=0$ can be up to double. (2) When the staggered potential satisfies $\lambda_{E}>\lambda_{\text {SO }}-M+V_{D S} / 2$, the edge state transformed from the quantum spin Hall state to the spin-polarized quantum anomalous Hall state, and the $K$ and $K^{\prime}$ valleys are $+100 \%$ or $-100 \%$ spin polarized in different energy ranges. Consequently, the FM germanene FET can be turned off. (3) The switching-off mechanism of the FM germanene FET is related to the electric field strength. For $\lambda_{E}<\lambda_{\text {SO }}$, the transistor in the off state is mainly due to spin blocking caused by the edge-state mismatch, for $\lambda_{E}>\lambda_{\text {So }}$, both the edgestate mismatch and the energy-valley mismatch are used. For $\lambda_{E}>2 \lambda_{\text {So }}$, besides the energy-valley mismatch, the $K$ and $K^{\prime}$ valley gaps are also used, and the subthreshold swing can be reduced to half that of the dual-channel normal germanene FET. (4) The strength of the exchange field in the range of $0<M<\lambda_{\text {so }}$ does not change the switching mechanism. However, the stronger the exchange field is, the lower the threshold electric field required for the off state is, and the higher the breakdown voltage of the transistor becomes.

\section{ACKNOWLEDGMENTS}

This work is supported by the National Natural Science Foundation of China (Grants No. 12174038 and No. 12074209), the LiaoNing Revitalization Talents Program (XLYC2007141), the Open Project of State Key
Laboratory of Low-Dimensional Quantum Physics (Grant No. KF201910), and the Science Technology Foundation from Education Commission of Beijing (Grant No. KM201810028022).

[1] K. Takeda and K. Shiraishi, Theoretical possibility of stage corrugation in Si and Ge analogs of graphite, Phys. Rev. B 50, 14916 (1994).

[2] L. D. Landau and E. M. Lifshitz, Statistical Physics, Part I. (Pergamon Press, Oxford, 1980).

[3] N. D. Mermin, Crystalline order in two dimensions, Phys. Rev. 176, 250 (1968).

[4] K. S. Novoselov, A. K. Geim, S. V. Morozov, D. Jiang, Y. Zhang, S. V. Dubonos, I. V. Grigorieva, and A. A. Firsov, Electric field effect in atomically thin carbon films, Science 306, 666 (2004).

[5] A. K. Geim and K. S. Novoselov, The rise of graphene, Nat. Mater. 6, 183 (2007).

[6] S. Lebegue and O. Eriksson, Electronic structure of twodimensional crystals from ab initio theory, Phys. Rev. B 79, 115409 (2009).

[7] M. Houssa, G. Pourtois, V. V. Afanasev, and A. Stesmans, Electronic properties of two-dimensional hexagonal germanium, Appl. Phys. Lett. 96, 082111 (2010).

[8] S. Cahangirov, M. Topsakal, E. Akturk, H. Sahin, and S. Ciraci, Two- and One-Dimensional Honeycomb Structures of Silicon and Germanium, Phys. Rev. Lett. 102, 236804 (2009).

[9] C. C. Liu, H. Jiang, and Y. Yao, Low-energy effective hamiltonian involving spin-orbit coupling in silicene and two-dimensional germanium and tin, Phys. Rev. B 84, 195430 (2011).

[10] N. D. Drummond, V. Zolyomi, and V. I. Falko, Electrically tunable band gap in silicene, Phys. Rev. B 85, 075423 (2012).

[11] M. Ezawa, A topological insulator and helical zero mode in silicene under an inhomogeneous electric field, New J. Phys. 14, 033003 (2012).

[12] W. F. Tsai, C. Y. Huang, T. R. Chang, H. Lin, H. T. Jeng, and A. Bansil, Gated silicene as a tunable source of nearly $100 \%$ spin-polarized electrons, Nat. Commun. 4, 1500 (2013).

[13] L. L. Tao, A. Naeemi, and E. Y. Tsymbal, Valley-Spin Logic Gates, Phys. Rev. Appl. 13, 054043 (2020).

[14] H. Chen, W. W. Li, Y. M. Chang, C. Y. Lin, S. H. Yang, Y. Xu, and Y. F. Lin, Negative-Differential-Resistance Devices Achieved by Band-Structure Engineering in Silicene under Periodic Potentials, Phys. Rev. Appl. 10, 044047 (2018).

[15] Z. Ni, Q. Liu, K. Tang, J. Zheng, J. Zhou, R. Qin, Z. Gao, D. $\mathrm{Yu}$, and J. Lu, Tunable bandgap in silicene and germanene, Nano Lett. 12, 113 (2012).

[16] M. Ezawa, Quantized conductance and field-effect topological quantum transistor in silicene nanoribbons, Appl. Phys. Lett. 102, 172103 (2013).

[17] S. Kaneko, H. Tsuchiya, Y. Kamakura, N. Mori, and M. Ogawa, Theoretical performance estimation of silicene, 
germanene, and graphene nanoribbon field-effect transistors under ballistic transport, Appl. Phys. Express 7, 035102 (2014).

[18] Z. Y. Ni, H. X. Zhong, X. H. Jiang, R. G. Quhe, G. F. Luo, Y. Y. Wang, M. Ye, J. B. Yang, J. J. Shi, and J. Lu, Tunable band gap and doping type in silicene by surface adsorption: Towards tunneling transistors, Nanoscale, 6, 7609 (2014).

[19] X. C. Zhai and G. J. Jin, Completely independent electrical control of spin and valley in a silicene field effect transistor, J. Phys.: Condens. Matter 28, 355002 (2016).

[20] Y. Katayama, R. Yamauchi, Y. Yasutake, S. Fukatsu, and K. Ueno, Ambipolar transistor action of germanane electric double layer transistor, Appl. Phys. Lett. 115, 122101 (2019).

[21] J. Zheng, Y. Xiang, C. L. Li, R. Y. Yuan, F. Chi, and Y. Guo, All-Optically Controlled Topological Transistor Based on $X$ enes, Phys. Rev. Appl. 14, 034027 (2020).

[22] L. F. Li, S. Z. Lu, J. B. Pan, Z. H. Qin, Y. Q. Wang, Y. L. Wang, G. Y. Cao, S. X. Du, and H. J. Gao, Buckled germanene formation on Pt (111), Adv. Mater. 26, 4820 (2014).

[23] M. E. Davila, L. Xian, S. Cahangirov, A. Rubio, and G. LeLay, Two-dimensional dirac signature of germanene, New J. Phys. 16, 095002 (2014).

[24] L. Zhang, P. Bampoulis, A. van Houselt, and H. J. W. Zandvliet, Two-dimensional dirac signature of germanene, Appl. Phys. Lett. 107, 111605 (2015).

[25] M. Derivaz, D. Dentel, R. Stephan, M. C. Hanf, A. Mehdaoui, P. Sonnet, and C. Pirri, Continuous germanene layer on $\mathrm{Al}$ (111), Nano Lett. 15, 2510 (2015).

[26] L. Zhang, P. Bampoulis, A. N. Rudenko, Q. Yao, A. van Houselt, B. Poelsema, M. I. Katsnelson, and H. J. W. Zandvliet, Structural and Electronic Properties of Germanene on $\mathrm{MoS}_{2}$, Phys. Rev. Lett. 116, 256804 (2016).

[27] L. Tao, E. Cinquanta, D. Chiappe, C. Grazianetti, M. Fanciulli, M. Dubey, A. Molle, and D. Akinwande, Silicene field-effect transistors operating at room temperature, Nat. Nanotech. 10, 227 (2015).

[28] B. N. Madhushankar, A. Kaverzin, T. Giousis, G. Potsi, D. Gournis, P. Rudolf, G. RBlake, C. H. van der Wal, and B. J. van-Wees, Electronic properties of germanane field-effect transistors, 2D Mater. 4, 021009 (2017).

[29] M. Z. Hasan and C. L. Kane, Colloquium: Topological insulators, Rev. Mod. Phys. 82, 3045 (2010).

[30] X. L. Qi and S. C. Zhang, Topological insulators and superconductors, Rev. Mod. Phys. 83, 1057 (2011).

[31] X. W. Jiang and S. S. Li, Performance limits of tunnel transistors based on mono-layer transition-metal dichalcogenides, Appl. Phys. Lett. 104, 193510 (2014).

[32] C. L. Kane and E. J. Mele, $Z_{2}$ Topological Order and the Quantum Spin Hall Effect, Phys. Rev. Lett. 95, 146802 (2005).

[33] M. Ezawa, Valley-Polarized Metals and Quantum Anomalous Hall Effect in Silicene, Phys. Rev. Lett. 109, 055502 (2012).

[34] J. Zheng, F. Chi, and Y. Guo, Thermal Spin Generator Based on a Germanene Nanoribbon Subjected to Local Noncollinear Exchange Fields, Phys. Rev. Appl. 9, 024012 (2018).
[35] J. Zheng, F. Chi, and Y. Guo, Spin-current diodes based on germanene and stanene subjected to local exchange fields, Appl. Phys. Lett. 113, 112404 (2018).

[36] F. D. M. Haldane, Model for a Quantum Hall Effect Without Landau Levels: Condensed-Matter Realization of the "parity Anomaly", Phys. Rev. Lett. 61, 2015 (1988).

[37] C. L. Kane and E. J. Mele, Quantum Spin Hall Effect in Graphene, Phys. Rev. Lett. 95, 226801 (2005).

[38] Kh. Shakouri, H. Simchi, M. Esmaeilzadeh, H. Mazidabadi, and F. M. Peeters, Tunable spin and charge transport in silicene nanoribbons, Phys. Rev. B 92, 035413 (2015).

[39] H. Haugen, D. Huertas-Hernando, and A. Brataas, Spin transport in proximity-induced ferromagnetic graphene, Phys. Rev. B 77, 115406 (2008).

[40] T. Yokoyama, Controllable valley and spin transport in ferromagnetic silicene junctions, Phys. Rev. B 87, 241409(R) (2013).

[41] H. X. Yang, A. Hallal, D. Terrade, X. Waintal, S. Roche, and M. Chshiev, Proximity Effects Induced in Graphene by Magnetic Insulators: First-Principles Calculations on Spin Filtering and Exchange-Splitting Gaps, Phys. Rev. Lett. 110, 046603 (2013).

[42] S. Rachel and M. Ezawa, Giant magnetoresistance and perfect spin filter in silicene, germanene, and stanene, Phys. Rev. B 89, 195303 (2014).

[43] C. $\mathrm{Xu}$ and J. E. Moore, Stability of the quantum spin hall effect: Effects of interactions, disorder, and $Z_{2}$ topology, Phys. Rev. B 73, 045322 (2006).

[44] C. J. Wu, B. A. Bernevig, and S. C. Zhang, Helical Liquid and the Edge of Quantum Spin Hall Systems, Phys. Rev. Lett. 96, 106401 (2006).

[45] Y. Meir and N. S. Wingreen, Landauer Formula for the Current through an Interacting Electron Region, Phys. Rev. Lett. 68, 2512 (1992).

[46] S. Datta, Electronic Transport in Mesoscopic Systems (Cambridge University Press, New York, 1995).

[47] D. H. Lee and J. D. Joannopoulos, Simple scheme for surface-band calculations. I, Phys. Rev. B 23, 4988 (1981).

[48] D. H. Lee and J. D. Joannopoulos, Simple scheme for surface-band calculations. II. The green's function, Phys. Rev. B 23, 4997 (1981).

[49] A. P. Jauho, N. S. Wingreen, and Y. Meir, Timedependent transport in interacting and noninteracting resonant-tunneling systems, Phys. Rev. B 50, 5528 (1994).

[50] B. K. Nikolic, L. P. Zarbo, and S. Souma, Imaging mesoscopic spin hall flow: Spatial distribution of local spin currents and spin densities in and out of multiterminal spinorbit coupled semiconductor nanostructures, Phys. Rev. B 73, 075303 (2006).

[51] J. Li and S. Q. Shen, Spin-current-induced charge accumulation and electric current in semiconductor nanostructures with Rashba spin-orbit coupling, Phys. Rev. B 76, 153302 (2007).

[52] H. Jiang, L. Wang, Q. F. Sun, and X. C. Xie, Numerical study of the topological anderson insulator in $\mathrm{HgTe} / \mathrm{CdTe}$ quantum wells, Phys. Rev. B 80, 165316 (2009).

[53] Y. X. Xing, J. Wang, and Q. F. Sun, Focusing of electron flow in a bipolar graphene ribbon with different chiralities, Phys. Rev. B 81, 165425 (2010). 
[54] Y. X. Xing, L. Zhang, and J. Wang, Topological anderson insulator phenomena, Phys. Rev. B 84, 035110 (2011).

[55] H. Haug and A. P. Jauho, Quantum Kinetics in Transport and Optics of Semiconductors (Springer, Berlin, 2008).

[56] C. C. Liu, W. X. Feng, and Y. G. Yao, Quantum Spin Hall Effect in Silicene and Two-Dimensional Germanium, Phys. Rev. Lett. 107, 076802 (2011).
[57] D. Q. Zou, W. K. Zhao, C. F. Fang, B. Cui, and D. S. Liu, The electronic transport properties of zigzag silicene nanoribbon slices with edge hydrogenation and oxidation, Phys. Chem. Chem. Phys. 18, 11513 (2016).

[58] V. Sharma, P. Srivastava, and N. K. Jaiswal, Prospects of asymmetrically $\mathrm{H}$-terminated zigzag germanene nanoribbons for spintronic application, Appl. Surf. Sci. 396, 1352 (2017). 